\title{
THE BOUNDARY VALUES OF ANALYTIC FUNCTIONS*
}

\author{
BY
}

JOSEPH L. DOOB

Let $f(z)$ be a function analytic in the interior of the unit circle $|z|<1$. Then under certain conditions $\lim _{r=1} f\left(r e^{i t}\right)$ exists for almost all $t$ in $0 \leqq t<2 \pi$, defining a boundary function $F(z)$ almost everywhere on $|z|=1, z=e^{i t}$. The purpose of this paper is to discuss the function $F(z) . \dagger$

\section{The Ostrowski-NeVANLINNA TheOREM}

Let $\log ^{+} x=\frac{1}{2}(\log x+|\log x|)$. Ostrowski proved that if $f(z)$ is a function analytic in $|z|<1$, and if $\int_{0}^{2 \pi} \log ^{+}\left|f\left(r e^{i t}\right)\right| d t$ is bounded uniformly for $0 \leqq r<1$, then

$$
\lim _{r=1} f\left(r e^{i t}\right)=F\left(e^{i t}\right)
$$

exists for almost all $t$ in $0 \leqq t<2 \pi$ and $\int_{0}^{2 \pi} \log \left|F\left(e^{i t}\right)\right| d t$ exists. $\neq$

THEOREM 1. Let $f(z)$ be a function analytic in $|z|<1$, such that

$$
\int_{0}^{2 \pi} \psi\left\{\log +\left|f\left(r e^{i t}\right)\right|\right\} d t
$$

is bounded uniformly for $0 \leqq r<1$, where $\psi(x)$ is a real monotone non-decreasing function of $x$ such that $\lim _{x=\infty} \psi(x) / x=\infty$. Then $\lim _{r=1} f\left(r e^{i t}\right)$ exists for almost all $t, 0 \leqq t<2 \pi$, defining a boundary function $F\left(e^{i t}\right)$ such that $\int_{0}^{2 \pi} \log \left|F\left(e^{i t}\right)\right| d t$ exists and such that

$$
\log |f(0)| \leqq \frac{1}{2 \pi} \int_{0}^{2 \pi} \log \left|F\left(e^{i t}\right)\right| d t . \S
$$

We can assume that $f(0) \neq 0$ without loss of generality. Let

$$
L_{r}^{+}(f)=\frac{1}{2 \pi} \int_{0}^{2 \pi} \log ^{+}\left|f\left(r e^{i t}\right)\right| d t .
$$

* Preliminary report presented to the Society, April 3, 1931; received by the editors June 22' 1931.

† I wish to express my thanks to Professor J. L. Walsh and Dr. W. Seidel with whom I discussed the problems considered.

$\ddagger$ A. Ostrowski, Acta Litterarum ac Scientiarum Szeged, vol. 1 (1922), pp. 80-83; see also F. and R. Nevanlinna, Acta Societatis Scientiarum Fennicae, vol. 50, No. 5 (1922), p. 26, and F. Riesz, Acta Litterarum ac Scientiarum Szeged, vol. 1 (1922), pp. 95-96.

$\S$ If $f(0)=0$ take $\log f(0)=-\infty$. Theorem 1 was proved first with the hypothesis that $\int_{0}^{2 \pi}\left|f\left(r e^{i t}\right)\right| \delta d t, \delta>0$, was bounded uniformly for $0 \leqq r<1$. Professor J. D. Tamarkin suggested this proof, which follows a method of V. Smirnoff, Journal of the Leningrad Physico-Mathematical Society, vol. 2 (1929), pp. 34-35. The theorem first proved (by a different method) is obtained by setting $\Psi(x)=e^{\delta x}$. 
Since $\lim _{x=\infty} \psi(x) / x=\infty$, and since

$$
\int_{0}^{2 \pi} \psi\left\{\log ^{+}\left|f\left(r e^{i t}\right)\right|\right\} d t
$$

is bounded uniformly for $0 \leqq r<1, L_{r}^{+}(f)$ must also be bounded uniformly for $0 \leqq r<1$. Then, using the results of Ostrowski stated above, only the proof of the inequality (1) remains to be given. It follows from the Jensen-Nevanlinna formula* that

$$
\log |f(0)| \leqq L_{r}^{+}(f)-L_{r}^{+}(1 / f) \text {. }
$$

To prove the theorem it is therefore sufficient to show that

$$
\lim _{r=1} L_{r}^{+}(f)=L^{+}(F)=\frac{1}{2 \pi} \int_{0}^{2 \pi} \log ^{+}\left|F\left(e^{i t}\right)\right| d t
$$

and that

$$
\lim _{r=1} L_{r}^{+}(1 / f) \geqq L^{+}(1 / F) .
$$

Since $\log ^{+}\left|f\left(r e^{i t}\right)\right| \geqq 0$ and since

$$
\int_{0}^{2 \pi} \psi\left\{\log ^{+}\left|f\left(r e^{i t}\right)\right|\right\} d t
$$

is bounded uniformly for $0 \leqq r<1$, (A) is a consequence of a theorem of de la Vallée Poussin. $\dagger$ Since

$$
\log ^{+}\left(\frac{1}{\left|f\left(r e^{i t}\right)\right|}\right) \geqq 0,
$$

(B) is a consequence of Fatou's lemma.f The theorem is thus completely proved.

The following corollary is well known, in a somewhat different form and under other hypotheses. $\S$

Corollary 1. Let $f(z)$ be a function analytic for $|z|<1$ such that

$$
\int_{0}^{2 \pi}\left|f\left(r e^{i t}\right)\right|^{\delta} d t, \delta>0,
$$

* See for instance L. Bieberbach, Lehrbuch der Funktionentheorie, vol. 2, 2d edition, 1931, pp. $122-23$

† C. de la Vallee Poussin, these Transactions, vol. 16 (1915), p. 451.

$\ddagger$ P. Fatou, Acta Mathematica, vol. 30 (1906), pp. 375-76.

§ Ostrowski, loc. cit., p. 86; F. and R. Nevanlinna, loc. cit., pp. 11-12. 
is bounded uniformly for $0 \leqq r<1$. Let $E_{1}, \cdots, E_{n}$ be mutually exclusive measurable point sets on $|z|=1$ such that $m E_{j}>0^{*}, j=1, \cdots, n$, and such that $m E_{1}+\cdots+m E_{n}=2 \pi$. Then if $\dagger$

$$
\left\{\frac{1}{m E_{j}} \int_{E_{j}}|F(z)|^{\delta}|d z|\right\}^{1 / 8} \leqq \eta_{j} \quad(j=1, \cdots, n),
$$

it follows that

$$
|f(0)| \leqq \prod_{j=1}^{n} \eta_{j}{ }^{m E_{j} /(2 \pi)}
$$

It will be noted that the $k$ th condition of (3) is satisfied if $|F(z)| \leqq \eta_{j}$ almost everywhere on $E_{j}$. Corollary 1 is obvious if $f(0)=0$. If $f(0) \neq 0$, the corollary is deduced from Theorem 1 by means of a well known integral inequality: if $g(x)$ is a real not-negative integrable function of $x$ on a measurable set $E$ such that $\int_{E} \log g(x) d x$ exists, then

$$
\log \frac{1}{m E} \int_{E} g(x) d x \geqq \frac{1}{m E} \int_{E} \log g(x) d x . \ddagger
$$

If we set $g(t)=\left|F\left(e^{i t}\right)\right|^{\delta}$ we find that

$$
\begin{aligned}
\frac{1}{m E_{j}} \int_{E_{j}} \log |F(z)||d z|=\frac{1}{\delta m E_{j}} \int_{E_{j}} \log \left|F\left(e^{i t}\right)\right|^{\delta} d t & \\
& \leqq \frac{1}{\delta} \log \frac{1}{m E_{j}} \int_{E_{j}}|F(z)|^{\delta}|d z|,
\end{aligned}
$$

so that

$$
\log |f(0)| \leqq \frac{1}{2 \pi} \int_{|z|=1} \log |F(z)||d z| \leqq \frac{1}{2 \pi} \sum_{j=1}^{n} m E_{j} \log \eta_{j}
$$

which is equivalent to the inequality (4).

* $m E_{j}$ denotes the Lebesgue measure of $E_{j}$.

† F. Riesz, Mathematische Zeitschrift, vol. 18 (1923), pp. 87-95, proved that under the hypotheses stated here

$$
\lim _{r-1} f\left(r e^{i t}\right)=F\left(e^{i t}\right)
$$

exists for almost all $t, 0 \leqq t<2 \pi$, and that

exists.

$$
\int_{\mid z-1}|F(z)|^{\delta}|d z|=\lim _{r-1} \int_{|z|-r}|f(z)|^{\delta}|d z|
$$

$\ddagger$ This inequality can easily be obtained from a generalized inequality between arithmetic and geometric means, for which see G. Polya and G. Szegö, Aufgaben und Lehrsätze aus der Analysis, vol. I, p. 53, Aufgabe 78 . 
CoRollary 2. If in Corollary 1 the inequalities (3) are replaced by

$$
|F(z)| \leqq \eta_{j},
$$

for $z$ almost everywhere on $E_{j}, j=1, \cdots, n$, it follows that

$$
|f(z)| \leqq \prod_{j=1}^{n} \eta_{i} P_{i}(z)
$$

where $P_{j}(z)$ is the harmonic function defined by

$$
P_{j}(z)=\frac{1}{2 \pi} \int_{0}^{2 \pi} U_{j}\left(e^{i t}\right) \frac{1-r^{2}}{1-2 r \cos (\psi-t)+r^{2}} d t, z=r e^{i \psi},
$$

$U_{j}(z)=1$ for $z$ in $E_{j}, U_{j}(z)=0$ on the complementary set.

The proof of this is simply the application of Corollary 1 to the function

$$
f_{1}(w)=f\left(\frac{w-z}{\bar{z} w-1}\right){ }^{*}
$$

a function which is analytic in $|w|<1$ and has the value $f(z)$ at $w=0$. This result will be the basis for the discussion of §III in which functions of the form $P_{j}(z)$ will be considered in some detail. $2 \pi P_{j}(z)$ is the measure of the point set into which $E_{j}$ is transformed by the linear transformation

$$
w^{\prime}=\frac{w-z}{\bar{z} w-1} .
$$

Corollary 3. Let $f(z), F(z)$ be the same as in Theorem 1 and suppose in addition that $f(z) \not \equiv 0, f(z)=\alpha z^{k}+\cdots, \alpha \neq 0, k \geqq 0$. Then if $E_{\mathrm{c}}$ is the set of those points on $|z|=1$ for which $|F(z)| \leqq \epsilon, m E_{\mathrm{\epsilon}}$ has an upper bound approaching 0 with $\epsilon$ and depending only on $\epsilon,|\alpha|, M$, where $\log M \geqq L^{+}(f), 0 \leqq r<1$.

As noted in the proof of Theorem 1, a constant $M$ such that $\log M \geqq L_{r}^{+}(f)$, $0 \leqq r<1$, actually exists. Let $f_{0}(z)=f(z) / z^{k}$. It is a simple matter to show that there exists a real monotone non-decreasing function $\psi_{0}(x)$ such that

and such that

$$
\lim _{x=\infty} \psi_{0}(x) / x=\infty
$$

$$
\int_{0}^{2 \pi} \psi_{0}\left\{\log ^{+}\left|f_{0}\left(r e^{i t}\right)\right|\right\} d t
$$

is bounded uniformly for $0 \leqq r<1$. It is almost obvious that

$$
\limsup _{r=1} L_{r}^{+}\left(f_{0}\right) \leqq \log M \text {. }
$$

$* \bar{z}$, as is customary, is the conjugate complex number of $z$. 
Leaving these facts for the reader to verify, we proceed with the proof. We have

(7) $L^{+}(1 / F) \leqq \lim _{r=1} \inf _{r}^{+}\left(1 / f_{0}\right) \leqq \liminf _{r=1} L_{r}^{+}\left(f_{0}\right)-\log |\alpha| \leqq \log (M /|\alpha|)$,

using the inequalities (B) and (2) obtained in the proof of Theorem 1. By the equality (A) above, and since

$$
\begin{gathered}
\left|\lim _{r=1} f_{0}\left(r e^{i t}\right)\right|=\left|F\left(e^{i t}\right)\right|, \\
L^{+}(F)=\lim _{r=1} L_{r}^{+}\left(f_{0}\right) \leqq \log M,
\end{gathered}
$$

so that combining inequalities (7) and (8),

$$
\frac{1}{2 \pi} \int_{0}^{2 \pi}|\log | F\left(e^{i t}\right)|| d t \leqq \log \left(M^{2} /|\alpha|\right) .
$$

It is now easy to prove Corollary 3. Apply Theorem 1 to $f_{0}(z)$ :

$$
\log |\alpha| \leqq \frac{1}{2 \pi} \int_{E_{\mathrm{c}}} \log |F(z)||d z|+\frac{1}{2 \pi} \int_{C E_{\mathrm{\epsilon}}} \log |F(z)||d z|
$$

where $C E_{\mathrm{c}}$ is the complementary set of $E_{\mathrm{c}}$ on $|z|=1$. Then using (9),

$$
\log |\alpha| \leqq \frac{1}{2 \pi} m E_{\epsilon} \log \epsilon+\log \left(M^{2} /|\alpha|\right) .
$$

so that if $\epsilon<1, \log \epsilon<0$,

If

$$
m E_{\epsilon} \leqq 4 \pi \frac{\log \left(\frac{|\alpha|}{M}\right)}{\log \epsilon} .
$$

$$
\int_{0}^{2 \pi}\left|f\left(r e^{i t}\right)\right|^{\delta} d t, \delta>0
$$

is bounded uniformly for $0 \leqq r<1$,

$$
\int_{0}^{2 \pi}\left|f\left(r e^{i t}\right)\right|^{\delta} d t \leqq N^{s}
$$

inequality (4) leads to

$$
m E_{\mathrm{\epsilon}} \leqq 2 \pi \frac{\log \left(\frac{c^{1 / 8}|\alpha|}{N}\right)}{\log \left(\frac{\epsilon}{N}\right)}, c=\left(\frac{1}{e}\right)^{1 / 0}
$$




\section{Metric Density AND MEtRiC Cluster VAlues}

Let a measurable point set $E$ be given on $|z|=1$. The set $E$ will be said to be metrically dense at a point $P$ (which may or may not belong to $E$ ) if every neighborhood of $P$ contains a subset of $E$ of positive measure. This concept is made more precise as follows. If $A$ is an $\operatorname{arc}$ (open or closed) with $P$ as midpoint, the inferior and superior limits of $m(E \cdot A) / m A$ as $m A$ approaches 0 will be called the lower and upper mean metric densities of $E$ at $P$ respectively. ${ }^{*}$ If these are equal, their common value will be called the mean metric density of $E$ at $P$. If the mean metric density of $E$ exists and is 1 at a point $P$, the metric density of $E$ exists and is 1 at $P$ and conversely. By a theorem of Lebesgue $\dagger$ the metric density of $E$ exists and is 1 almost everywhere in $E$, which implies the same fact for the mean metric density.

Let $E$ be a measurable point set on the $x$-axis. Then the lower metric density of $E$ on the right at $x=x_{0}$ is defined as

$$
\liminf _{m I=0} \frac{m(E \cdot I)}{m I}
$$

where $I$ is an interval lying on the right of $x_{0}$ and having $x_{0}$ as one end point.

Lеммa 2.1. Let $E, E^{\prime}$ be measurable point sets on the interval $0 \leqq x \leqq 1$, having lower metric density $\delta, \delta^{\prime}$ respectively on the right at $x=0$. Let the points of $E$ be transformed into those of $E^{\prime}$ in a one-to-one way by the transformation $x^{\prime}=\psi(x)$, with inverse $x=\psi_{1}\left(x^{\prime}\right)$, where $\psi(x), \psi^{\prime}(x)$ are continuous and not negative in $0 \leqq x \leqq 1$ and $\psi(0)=0$. Then if $\psi^{\prime}\left(0^{+}\right)>0, \delta^{\prime}=\delta$. If $\psi(x)=x^{\nu}, \nu>1$,

$$
\delta^{\prime} \geqq\left(\frac{\nu-1}{\nu}\right)^{\nu-1} \delta^{\nu} .
$$

Let $\phi(x)=1$ if $x$ belongs to the set $E$, and 0 otherwise; then

and

$$
\delta=\liminf _{y=0} \frac{1}{y} \int_{0}^{y} \phi(x) d x
$$

$$
\delta^{\prime}=\liminf _{y=0} \frac{1}{y} \int_{0}^{y} \phi\left[\psi_{1}(x)\right] d x
$$

or, since $\psi(0)=0$,

$$
\delta^{\prime}=\liminf _{y=0} \frac{1}{\psi(y)} \int_{0}^{\psi(y)} \phi\left[\psi_{1}(x)\right] d x=\liminf _{y=0} \frac{1}{\psi(y)} \int_{0}^{\nu} \phi(x) \psi^{\prime}(x) d x .
$$

* The lower and upper mean metric densities as defined here are not the same as the lower and upper metric densities, for a discussion of which see Hobson, The Theory of Functions of a Real Variable, vol. 1, 2d edition, 1921, pp. 178-182.

$\dagger$ H. Lebesgue, Annales de l'Ecole Normale, (3), vol. 27 (1910), p. 407. 
If $\psi^{\prime}\left(0^{+}\right)=a>0$,

$$
\delta^{\prime}=\liminf _{y=0}\left\{\frac{a}{\psi(y)} \int_{0}^{y} \phi(x) d x+\frac{1}{\psi(y)} \int_{0}^{y} \phi(x)\left[\psi^{\prime}(x)-a\right] d x\right\} .
$$

The last term has the limit 0 when $y$ approaches 0 since if $y_{0}$ is so small that (i) $\left|\psi^{\prime}(x)-a\right| \leqq \epsilon a$ when $0 \leqq x \leqq y_{0}$ and that (ii) $a y / \psi(y) \leqq 2,0<y \leqq y_{0}$,

$$
\left|\frac{1}{\psi(y)} \int_{0}^{y} \phi(x)\left[\psi^{\prime}(x)-a\right] d x\right| \leqq \frac{\epsilon a y}{\psi(y)} \leqq 2 \epsilon, 0<y \leqq y_{0} .
$$

Then

$$
\delta^{\prime}=\liminf _{y=0}\left\{\frac{a}{\psi(y)} \int_{0}^{y} \phi(x) d x\right\}=\liminf _{y=0}\left\{\left(\frac{a y}{\psi(y)}\right) \frac{1}{y} \int_{0}^{y} \phi(x) d x\right\}=\delta .
$$

If $\psi(x)=x^{\nu}, \nu>1$,

$$
\begin{aligned}
\delta^{\prime} & =\liminf _{y=0} \frac{\nu}{y^{\nu}} \int_{0}^{y} \phi(x) x^{\nu-1} d x \geqq \liminf _{y=0} \frac{\nu}{y^{\nu}} \int_{\lambda_{y}}^{y} \phi(x) x^{\nu-1} d x \\
& \geqq \liminf _{y=0} \frac{\nu \lambda^{\nu-1}}{y} \int_{\lambda_{y}}^{y} \phi(x) d x,
\end{aligned}
$$

where $0<\lambda<1$. Then

$$
\delta^{\prime} \geqq \liminf _{y=0}\left\{\nu \lambda^{\nu-1}\left(\frac{1}{y} \int_{0}^{y} \phi(x) d x\right)-\nu \lambda^{\nu-1}\left(\frac{1}{y} \int_{0}^{\lambda y} \phi(x) d x\right)\right\} \geqq \nu \delta \lambda^{\nu-1}-\nu \lambda^{\nu} .
$$

Setting $\lambda=((\nu-1) / \nu) \delta$, we get the desired result.

Let a measurable function $F(z)$ be defined almost everywhere on $|z|=1$. If the set of those points at which $\left|F(z)-F\left(z_{0}\right)\right| \leqq \epsilon, z_{0}$ fixed, has metric density 1 at $z_{0}$ for all $\epsilon>0, F(z)$ is called approximately continuous at $z_{0}$ by Denjoy. Denjoy proved that $F(z)$ is approximately continuous almost everywhere and that a necessary and sufficient condition that $F(z)$ be approximately continuous at a point $P$ is that $F(z)$ be continuous at $P$ on a point set containing $P$ and having metric density 1 at $P$. In order to state in a simple way the theorems which are to be proved, the concept of approximate continuity will be generalized. If a number $\alpha$ and a point $P$ exist such that the set of those points at which $|F(z)-\alpha| \leqq \epsilon$ has lower (upper) mean metric density $\delta_{\epsilon}$ at $P$, where

$$
\lim _{\epsilon=0} \epsilon^{\left(\delta_{\epsilon}\right)^{d}}=0, d>0
$$

* A. Denjoy, Bulletin de la Société Mathématique de France, vol. 43 (1915), pp. 165-173. 
$\alpha$ will be called a metric cluster value of the first (second) kind of $F(z)$ at $P$. The upper limit (which may be $+\infty$ ) of exponents $d$ for which

$$
\lim _{\epsilon=0} \epsilon^{\left(\delta_{\epsilon}\right)^{d}}=0
$$

will be called the order of the cluster value. A special case is $\delta_{\epsilon} \geqq \delta>0$, which reduces to approximate continuity at $P$ if $\delta=1$ and if $F(P)=\alpha$. A related special case is that in which $F(z)$ is continuous at $P$ on a set of positive lower (upper) mean metric density at $P$. The condition

$$
\lim _{\epsilon=0} \epsilon^{\left(\delta_{\epsilon}\right)^{d}}=0
$$

restricts the rapidity of approach of $\delta_{\epsilon}$ to 0 with $\epsilon$. Since $F(z)$ is approximately continuous almost everywhere on $|z|=1$, it has metric cluster values of the first kind (and of infinite order) almost everywhere there.

III. LINEAR TRANSFORMations OF THE UNIT CIRCLE AND Fatou's THEOREM

LEMMA 3.1. Let $E$ be a measurable point set on $|z|=1$. Let $A$ be an arc of $|z|=1$ with midpoint $P: z=1$. Then

$$
w=\frac{z-r}{r z-1}, z=\frac{w-r}{r w-1}
$$

transforms $|z| \leqq 1$ into $|w| \leqq 1$ and if $E$ is transformed into $E(r)$,

$$
m E(r) \geqq 4 \operatorname{arc} \tan \theta(E, A, r) \text {, }
$$

where

(11) $\theta(E, A, r)=\left\{\left(\frac{1+r}{1-r}\right) \frac{\tan \left(\frac{m A}{4}\right)-\tan \left(\frac{m A-m(E \cdot A)}{4}\right)}{1+\left(\frac{1+r}{1-r}\right)^{2} \tan \left(\frac{m A}{4}\right) \tan \left(\frac{m A-m(E \cdot A)}{4}\right)}\right\}$.

If $m(E \cdot A)<m A$ and if $r$ is determined by

(12) $r=\frac{a-1}{a+1}, a=\frac{1+r}{1-r}=\left\{\tan \left(\frac{m A}{4}\right) \tan \left(\frac{m A-m(E \cdot A)}{4}\right)\right\}^{-1 / 2}$,

it follows that

$$
m E(r) \geqq 4 \arctan \left\{\frac{m(E \cdot A)}{m A} \frac{1}{2}\left(1-\frac{m(E \cdot A)}{m A}\right)^{-1}\right\} .
$$

For

$$
m E(r)=\int_{E}\left|\frac{d w}{d z}\right||d z|=\int_{E} \frac{1-r^{2}}{|r z-1|^{2}} d z\left|\geqq \int_{E \cdot A} \frac{1-r^{2}}{|r z-1|^{2}}\right| d z \mid .
$$


Now on $|z|=1,|r z-1|=|r-1 / z|$ has its minimum at $z=1$ and increases monotonely as $z$ goes from 1 to -1 on either of the semi-circles in the upper and lower half-planes. Then if $z=e^{i t}$ on $|z|=1, \quad a=\frac{1}{2}[m A-m(E \cdot A)]$, $b=\frac{1}{2} m A$,

$$
m E(r) \geqq 2 \int_{a}^{b} \frac{1-r^{2}}{\left|r e^{i t}-1\right|^{2}} d t=4 \arctan \theta(E, A, r)
$$

which is (11). If $m(E \cdot A)<m A$ and if $r$ is determined by (12),

$$
m E(r) \geqq 4 \arctan \left\{\frac{\tan \left(\frac{m A}{4}\right)-\tan \left(\frac{m A-m(E \cdot A)}{4}\right)}{2\left[\tan \left(\frac{m A}{4}\right) \tan \left(\frac{m A-m(E \cdot A)}{4}\right)\right]^{1 / 2}}\right\} .
$$

To deduce (13) from this we note that if $x_{1}, x_{2}$ are real and positive, $x_{1} \leqq x_{2}$ $<\pi / 2$ implies that

$$
\frac{\tan x_{1}}{\tan x_{2}} \leqq \frac{x_{1}}{x_{2}}
$$

COROLLARY. If the set $E$ has lower (upper) mean metric density $\delta_{l}\left(\delta_{u}\right)$ at $P$,

$$
\begin{aligned}
& \lim _{r=1} \inf m E(r) \geqq 4 \arctan \left[\frac{1}{2} \delta_{l}\left(1-\delta_{l}\right)^{-1 / 2}\right] \text { if } \delta_{l}<1 \text {, } \\
& \lim m E(r)=2 \pi \quad \text { if } \delta_{l}=1, \\
& r=
\end{aligned}
$$

$\lim \sup m E(r) \geqq 4 \arctan \left[\frac{1}{2} \delta_{u}\left(1-\delta_{u}\right)^{-1 / 2}\right]$ if $\delta_{u}<1$, $r=1$

$$
\limsup _{r=1} m E(r)=2 \pi \quad \text { if } \delta_{u}=1 .
$$

This is an immediate consequence of (13), since, in (12), if $m A \rightarrow 0, r \rightarrow 1$. As a first application we sketch a proof of Fatou's theorem that if $U\left(e^{i t}\right)$ is a bounded measurable function of $t, 0 \leqq t<2 \pi$,

$$
u(z)=\int_{0}^{2 \pi} U\left(e^{i t}\right) \frac{1-r^{2}}{1-2 r \cos (\psi-t)+r^{2}} d t, z=r e^{i \psi},
$$

is a harmonic function in $|z|<1$ and $\lim _{r=1} u\left(r e^{i t}\right)=U\left(e^{i t}\right)$ for almost all $t$, $0 \leqq t<2 \pi$. $^{*}$

The fact that $u(z)$ is harmonic in $|z|<1$ is well known. The proof that the boundary function is actually $U\left(e^{i t}\right)$ will be sketched in two steps.

* P. Fatou, Acta Mathematica, vol. 30 (1906), pp. 345-349. The restriction that $U\left(e^{i t}\right)$ be bounded is not necessary: it is sufficient that $U\left(e^{i t}\right)$ be Lebesgue-integrable. 
(i) Let $U\left(e^{i t}\right)=1$ on a measurable set $E$ on $|z|=1$ and 0 elsewhere. Then in the notation of Lemma 3.1, $u(r)=m E(r) /(2 \pi)$. It is at once evident from the corollary to the lemma that $\lim _{r=1} u\left(r e^{i t}\right)=1=U\left(e^{i t}\right)$ almost everywhere in $E$ (wherever the metric density of $E$ is 1 ). Substituting its complementary set for $E$ and $1-U\left(e^{i t}\right)$ for $U\left(e^{i t}\right)$ it is seen that $\lim _{r=1} u\left(r e^{i t}\right)=0=U\left(e^{i t}\right)$ almost everywhere in the complement of $E$.

(ii) Let $U\left(e^{i t}\right)$ be a measurable function taking on only a finite number of values. Then it is a finite sum of functions considered in (i) and so the theorem is also true in this case. The transition to any bounded measurable function can be carried out without difficulty.

It can be deduced almost at once from this that if $f(z)$ is a bounded analytic function in $|z|<1, \lim _{r=1} f\left(r e^{i t}\right)$ exists for almost all $t, 0 \leqq t<2 \pi$.

IV. Metric Cluster VAlues of BOUNDED ANALYTIC FUNCTIONS IN THE UNIT CIRCLE AND NON-TANGENTIAL APPROACH TO THE PERIMETER

THEOREM 2. Let $f(z)$ be a bounded analytic function in $|z|<1$, with the boundary function $F(z),|f(z)| \leqq 1$. Let $E$ be a measurable point set on $|z|=1$ having lower (upper) mean metric density $\delta_{l}\left(\delta_{u}\right)$ at a point $P: e^{i t_{0}}$. Then if $|F(z)| \leqq \eta<1$ on $E$,

$$
\begin{aligned}
& \limsup _{r=1}\left|f\left(r e^{i t_{0}}\right)\right| \leqq \eta^{\sigma\left(\delta_{l}\right)}, \\
& \underset{r=1}{\lim \inf }\left|f\left(r e^{i t_{0}}\right)\right| \leqq \eta^{\sigma\left(\delta_{u}\right)},
\end{aligned}
$$

where

$$
\sigma(\delta)=\frac{2}{\pi} \arctan \left[\frac{\delta}{2}(1-\delta)^{-1 / 2}\right],
$$

if $\delta<1, \sigma(1)=1$.

We can suppose that $t_{0}=0$. Let $E$ become $E(r)$ under the transformation $w=(z-r) /(r z-1)$. Then as in Theorem 1, Corollary 2, we apply Theorem 1 to the function $f((z-r) /(r z-1))$, getting

$$
|f(r)| \leqq \eta^{m E(r) /(2 \pi)} 1^{1-m E(r) /(2 \pi)}=\eta^{m E(r) /(2 \pi)} .
$$

The theorem is now an immediate consequence of the corollary to Lemma 3.1.

We now define different types of cluster values of $f(z)$ at its boundary points. The point $z$ will be said to approach a point $P$ on $|z|=1$ on a nontangential path if $z$ remains within some angle whose vertex is at $P$ and whose sides are chords of $|z|=1$. Since we are only considering bounded functions, a sufficient condition that $f^{\prime}(z)$ have the limit $\alpha$ when $z$ approaches $P$ on any non-tangential path is that $f(z)$ have the limit $\alpha$ at $P$ when $z$ approaches $P$ on 
a single such path which is a simple Jordan arc.* $f(z)$ will be said to have the cluster value $\alpha$ when $z$ approaches a point $P$ of $|z|=1$ on some given path if there is a sequence of points $z_{1}, z_{2}, \cdots$, on the path such that $\lim _{n=\infty} f\left(z_{n}\right)=\alpha$, $z_{n} \rightarrow P$. We shall need a slight extension of the idea of metric cluster value as defined above. Let $f(z)$ have the cluster value $\alpha$ on a straight line $L$ to $P$ on $|z|=1$. Then if the set of points on $L$ at which $|f(z)-\alpha| \leqq \epsilon$ has lower (upper) metric density $\delta_{\epsilon}$ at $P$ on the side of $L$ within $|z|<1$, such that

$$
\lim _{\epsilon=0} \epsilon^{\left(\delta_{\epsilon}\right) d}=0, d>0,
$$

$\alpha$ will be called a metric cluster value of the first (second) kind of $f(z)$ on $L$ at $P$. The order is defined as before. If $F(z)$ is a measurable function defined almost everywhere on $|z|=1$, its metric cluster values on one side of a point on $|z|=1$ are defined in an obvious way. It is evident, however, from the symmetry of the definition of mean metric density, that a metric cluster value of $F(z)$ on one side of a point on $|z|=1$ is also a metric cluster value of $F(z)$ at the point as originally defined, and that the kind and order are unchanged.

TheOREM 3. Let $f(z)$ be a bounded analytic function in $|z|<1$ with the boundary function $F(z)$. Let $P$ be a point of $|z|=1$.

(i) The meiric cluster values of the first and second kinds of $F(z)$ at $P$ of order greater than 1 are contained in the set of cluster values of $f(z)$ on any single straight line to $P$.

(ii) If the set of metric cluster values of the first and second kinds of $F(z)$ at $P$ of order greater than 1 contains one of the first kind, $\alpha, f(z)$ has the unique limit $\alpha$ as $z$ approaches $P$ on non-tangential paths: $F(P)=\alpha$, and all the metric cluster values of the first two kinds of $F(z)$ at $P$ of order greater than 1 have the common value $\alpha$.

This generalizes a theorem proved by Pringsheim, Lindelöf and others which says that $F(z)$ cannot have a discontinuity of the first kind (a jump). For if $F(z)$ has the limit $\alpha$ on one side of $P, \beta$ on the other, $\alpha, \beta$ are limits on sets of mean metric density $\frac{1}{2}$. They are then both metric cluster values of the first kind (of infinite order), so, by (ii), $\alpha=\beta$. It is to be noted that the theorem does not state a necessary condition on $F(z)$ in order that $f(z)$ have the unique limit $\alpha$ when $z$ approaches $P$ on non-tangential paths. In the latter

* E. Lindelöf, Acta Societatis Scientiarum Fennicae, vol. 46, No. 4 (1915), p. 10. The theorem used, which will be generalized below, can be stated as follows. Let $f(z)$ be analytic and bounded in the region $0<r<r_{0}, r_{0}>0,|\phi|<\phi_{0}, \phi_{0}>0$, where $z=r e^{i \phi}$. Then if $\lim _{z=0} f(z)=\alpha$ when $z$ approaches $z=0$ on a simple Jordan arc lying in $0<r<r_{0},|\phi| \leqq \phi_{1}<\phi_{0}, \lim _{z=0} f(z)=\alpha$ uniformly in any region $0<r<r_{0},|\phi| \leqq \phi_{2}<\phi_{0}$. 
event, however, by (i), $F(z)$ can have no metric cluster values of the first two kinds of order greater than 1 other than $\alpha$.

The proof is a simple application of Theorem 2. Let $F(z)$ have $\alpha$ as a metric cluster value of the second kind of order greater than 1 at $P$. We can suppose that $P$ is the point $z=1$, that $\alpha=0$, and that $|F(z)| \leqq 1$. Then if the set of those points on $|z|=1$ at which $|F(z)| \leqq \epsilon$ has upper mean metric density $\delta_{\epsilon}$,

Since

$$
\liminf _{r=1}|f(r)| \leqq \epsilon^{\sigma\left(\delta_{e}\right)}
$$

$$
\lim _{\epsilon=0} \epsilon^{\delta_{\epsilon}}=0,
$$

by the definition of a metric cluster value of the second kind of order greater than 1 , and since

$$
\lim _{x=0} \frac{\arctan x}{x}=1
$$

for real $x$, we have

$$
\underset{r=1}{\lim \inf }|f(r)|=0
$$

on letting $\epsilon$ approach 0 . The value $\alpha=0$ must then be a cluster value of $f(z)$ on the radius to $P$. It will be shown in $\S \mathrm{V}$ that $\alpha$ is a cluster value of $f(z)$ when z. approaches $P$ on any chord of $|z|=1$. If $\alpha=0$ is a metric cluster value of the first kind of $F(z)$ at $P: z=1$ of order greater than 1, it is shown similarly that

$$
\limsup _{r=1}|f(r)|=\lim _{r=1} f(r)=0 .
$$

These two facts together with the theorem of Lindelöf quoted above in the note are sufficient to prove Theorem 3.

The following theorem generalizes the theorem of Lindelöf just used.

THEOREM 4. Let $f(z)$ be a bounded analytic function in $|z|<1$, and let $P$ be a point of $|z|=1$. Then if $f(z)$ has the metric cluster value $\alpha$ of the first kind of order greater than 2 on a single straight line to $P, \lim _{z \rightarrow P} f(z)=\alpha$ when $z$ approaches $P$ on any non-tangential path: $F(P)=\alpha$.

It is thus seen that for $f(z)$ to have a unique limit on a line to $P$ is the same as for it to have merely a metric cluster value of the first kind of order greater than 2 on the line at $P$. It will be seen from the discussion to be given that more general curves than straight lines could be used in the hypothesis, defining metric cluster values on these curves suitably. Theorem 4 evidently generalizes the theorem of Lindelöf given in the footnote on page 163, and 
can be stated in a similar way, for functions defined in a sector instead of in a circle.

The proof is an application of Theorem 3. Let $f(z)$ have the metric cluster value $\alpha$ of the first kind of order greater than 2 on the line $L$ to $P$. There is another chord $L^{\prime}$ of $|z|=1$ meeting $L$ at $P$ and forming with $L$ an angle of size $\tau=\pi /\left[2\left(1+\epsilon_{0}\right)\right]$ where $\epsilon_{0}>0$ is arbitrarily small. We consider $f(z)$ defined in the sector $S$ determined by $L, L^{\prime}$ and an arc of the circle of radius 1 , center at $P: z_{0}$. The sector $S$ can be transformed into a circle in the following way. First transform it into a semicircle by $z^{\prime}=\left(z-z_{0}\right)^{2\left(1+\epsilon_{0}\right)}$. The semicircle is then inverted on an end point of its bounding diameter, and thus transformed into a quadrant of the plane. The quadrant is transformed into a half-plane by a transformation of the form $z^{\prime}=\left(z-z_{1}\right)^{2}$ and the half-plane into a circle by a linear transformation. The product of all these transformations, $w=\phi(z)$, is a function analytic in $S$ in a neighborhood of $P$. The function $\phi(z)$ is compounded of functions with non-vanishing derivatives at $P$ and one of the form $z^{\prime}=\left(z-z_{0}\right)^{2\left(1+\epsilon_{0}\right)}$. Then if $E$ is a point set on $L$ in $|z|<1$ which is transformed into $E^{\prime}$ on $|w|=1$ by $w=\phi(z)$, if $E$ has lower metric density $\delta$ on $L$ at $P$ on the side in $|z|<1$, and if $E^{\prime}$ has lower metric density $\delta^{\prime}$ on $|w|=1$ at $P^{\prime}: \phi(P)$ on the corresponding side, $\delta^{\prime} \geqq c \delta^{2\left(1+\epsilon_{0}\right)}$, where

$$
c=\left(\frac{1+2 \epsilon_{0}}{2+2 \epsilon_{0}}\right)^{1+2 \epsilon_{0}}
$$

by Lemma 2.1. Let $z=\phi_{1}(w)$ be the branch of the inverse of $\phi(z)$ analytic in $|w|<1$ and taking it into the interior of $S$. Then if $f(z)$ has $\alpha$ as a metric cluster value of the first kind on $L$ at $P$ of order greater than 2 , the function $f\left[\phi_{1}(w)\right]$, bounded and analytic in $|w|<1$, has a boundary function on $|w|=1$ which has $\alpha$ as a metric cluster value of the first kind at $P^{\prime}$. Since $\epsilon_{0}$ can be made small at pleasure it is clear that the order of the cluster value at $P^{\prime}$ is greater than 1 . Then $f\left[\phi_{1}(w)\right]$ has the limit $\alpha$, by Theorem 4 , (ii), on all nontangential paths to $P^{\prime}$ so that $f(z)$ has the same limit on certain paths to $P$ in the sector $S$. By the theoren of Lindelöf (see footnote on page 163), $f(z)$ then has the limit $\alpha$ on all no i-tangential paths to $P$.

It is seen from the abovf proof that the order of the cluster value on $L$, instead of being taken greater than 2 could have been made dependent on the angle $\theta$ between $L$ and the radius to $P$. If $\theta$ is nearly $\pi / 2$ the order can be taken near 1.

If Theorems 3 and 4 are stated for functions analytic in a sector of a circle, approach to the vertex $P$ being considered, the only essential difference in statement is the difference in the orders of the metric cluster values considered. 
V. Metric Cluster values of bounded analytic functions in the UNit CIRCLE AND TANGENTIAL APPROACH TO THE PERIMETER

The discussion given in $\S I V$ has been restricted to approach to points of $|z|=1$ on non-tangential paths and even approach on non-tangential paths other than radii has not been treated directly. The methods used will now be applied directly to non-tangential (other than radial) approach and also to tangential approach to $|z|=1$. Part of Theorem 3 remains to be proved, in this connection. Let $f(z)$ be a bounded analytic function in $|z|<1$ with the boundary function $F(z)$. Let $F(z)$ have $\alpha$ as a metric cluster value of the second kind of order greater than 1 at $P$ on $|z|=1$. It was proved that $\alpha$ is then a cluster value at $P$ of $f(z)$ on the radius to $P$ and there remains the proof that $\alpha$ is a cluster value of $f(z)$ at $P$ on every other straight line to $P$. Let the point $\zeta$ trace such a straight line $L$. Let $E_{\epsilon}$ be the set of those points on $|z|=1$ at which $|F(z)-\alpha| \leqq \epsilon$ and let $E_{\epsilon}$ have upper mean metric density $\delta_{\epsilon}$ at $P$. We can suppose that $P$ is the point $z=1$, that $\alpha=0$, and that $|f(z)| \leqq 1$. Then let $E_{\epsilon}$ be transformed into $E_{\epsilon}\left(\zeta^{\zeta}\right)$ by the transformation

$$
w=\frac{z-\zeta}{\bar{\zeta} z-1} .
$$

Applying Theorem 1, Corollary 1, to $f((z-\zeta) /(\bar{\zeta} z-1))$,

$$
|f(\zeta)| \leqq \epsilon^{(1 /(2 \pi)) m E_{\epsilon}(\zeta)}
$$

If we can show that

$$
\limsup _{\zeta=1} m E_{\epsilon}(\zeta) \geqq \sigma_{1}\left(\delta_{\epsilon}\right) \text { where } \lim _{\delta=0} \sigma_{1}(\delta) / \delta=\kappa>0,
$$

we will have

$$
\liminf _{\zeta=1}|f(\zeta)| \leqq \epsilon^{(1 /(2 \pi)) \sigma_{1}\left(\delta_{\epsilon}\right)} .
$$

This, on letting $\epsilon$ approach 0 , becomes

$$
\liminf _{\zeta=1}|f(\zeta)|=0 \text {, since } \lim _{\epsilon=0} \epsilon_{\epsilon}^{\delta_{\epsilon}}=0,
$$

by the definition of metric cluster values of the second kind of order greater than 1. It will be shown, then, that if $\zeta$ is on $L$,

$$
\limsup _{\zeta=1} m E_{\epsilon}(\zeta) \geqq \sigma_{1}\left(\delta_{\epsilon}\right) \text { where } \lim _{\delta=0} \sigma_{1}(\delta) / \delta=\kappa>0 .
$$

Let the radius through $\zeta$ meet $|z|=1$ in $Q: e^{i t}$ and let $A$ be the arc of $|z|=1$ of length $4 t$ with midpoint $Q$. Then by Lemma 3.1, 


$$
\begin{aligned}
m E_{\epsilon}(\zeta) \geqq 4 \arctan \theta\left(E_{\epsilon}, A,|\zeta|\right) & \geqq 4 \arctan \left\{\left(\frac{1+|\zeta|}{1-|\zeta|}\right) \tan \left(\frac{m A}{4}\right) \frac{m\left(E_{\epsilon} \cdot A\right)}{m A}\right. \\
\cdot & {\left.\left[1+\left(\frac{1+|\zeta|}{1-|\zeta|}\right)^{2} \tan ^{2} \frac{m A}{4}\left(1-\frac{m\left(E_{\epsilon} \cdot A\right)}{m A}\right)\right]^{-1}\right\} }
\end{aligned}
$$

using the same argument as on page 161. Now since $\zeta=|\zeta| e^{i t}$ is on $L$, $|t| /(1-|\zeta|)$ has a limit $b$. Since $L$ is not the radius to $P: z=1, b>0$. Because of the choice of $A$,

$$
\limsup _{m A=0} \frac{m\left(E_{\epsilon} \cdot A\right)}{m A} \geqq \frac{1}{2} \delta_{\epsilon} .
$$

Then letting $m A$ approach 0 in (15),

$$
\limsup _{\zeta=1} m E_{\epsilon}(\zeta) \geqq 4 \arctan \left\{\frac{b \delta_{\epsilon}}{1+4 b^{2}\left(1-\frac{1}{2} \delta_{\epsilon}\right)}\right\} \geqq 4 \arctan \left(\frac{b \delta_{\epsilon}}{1+4 b^{2}}\right) .
$$

We take

$$
\sigma_{1}\left(\delta_{\epsilon}\right)=4 \arctan \left(\frac{b \delta_{\epsilon}}{1+4 b^{2}}\right)
$$

This satisfies the requirements since

$$
\lim _{\delta=0} \sigma_{1}(\delta) / \delta=\frac{4 b}{1+4 b^{2}}>0 .
$$

The desired result is thus proved, and from the proof as here given it is clear that the result could be stated in a stronger way.

We now take up tangential approach to $|z|=1$. Let $f(z)$ be a bounded analytic function in $|z|<1$ with boundary function $F(z)$. If $f(z)$ has the $\mathrm{u}$ nique limit $\alpha$ as $z$ approaches a point $P$ on $|z|=1$ on every curve tangent to $|z|=1$ at $P, F(z)$ must be continuous at $P$ (excluding from consideration the set of points of measure zero on $|z|=1$ at which $F(z)$ is not defined). For it follows readily from a theorem of Lindelöf* that $f(z)$ then also has the limit $\alpha$ on every path to $P$. Conversely if $F(z)$ is continuous at $P$ with value $\alpha$, possibly excluding from consideration a set of points on $|z|=1$ of zero measure, $\lim _{z \rightarrow P} f(z)=\alpha$ no matter how $z$ approaches $P$ from within $|z|=1$ by the same theorem of Lindelöf. The theorems which follow show the connection between cluster values of $f(z)$ at $P$ on curves tangent to $|z|=1$ at $P$ and the

* E. Lindelöf, Acta Societatis Scientiarum Fennicae, vol. 46, No. 4 (1915), p. 13. Theorem 3, (ii), is a generalization of this theorem. 
metric cluster values of $F(z)$ at $P$. The conditions on $F(z)$ need only be conditions on one side of $P$ since tangential approach to $P$ concerns only one side of the radius to $P$. Thus if $F(z)$ is continuous on one side of $P$ (excluding possibly from consideration a set of points on $|z|=1$ of measure zero), with limit value $\alpha, \lim _{z \rightarrow P} f(z)=\alpha$ when $z$ approaches $P$ on non-tangential paths, by Theorem 3, (ii). Using conformal mapping, it follows from the remarks just made concerning the conduct of a bounded analytic function in the neighborhood of a point of continuity of its boundary function, that $\lim _{z \rightarrow P} f(z)=\alpha$ for any manner of approach to $P$ as long as $z$ remains in the semicircle concerned: the one determined by the diameter through $P$ and on the side of the arc on which $F(z)$ has the limiting value $\alpha$ at $P$. The conditions to be set are then conditions on $F(z)$ at one side of $P$ and should imply less than continuity.

TheOREM 5. Let $f(z)$ be a bounded analytic function in $|z|<1,|f(z)| \leqq 1$, with the boundary function $F(z)$. Let $E$ be a point set on $|z|=1$ in the upper halfplane. If $A$ is an arc of $|z|=1$ in the upper half-plane with one end point at $P$ : $z=1$, let

$$
\limsup _{m A=0}\left[\frac{1-\frac{m\left(E_{\epsilon} \cdot A\right)}{m A}}{m A^{q} \cdot}\right]=\rho
$$

be finite, where $q>0$. Then if $|F(z)| \leqq \eta<1$ on $E$ and if the sequence $\left\{P_{n}\right.$ : $\left.r_{n} e^{i t_{n}}\right\}, 0 \leqq t_{n}<\pi, \lim _{n=\infty} P_{n}=P$, approach $P$ in such a way that

$$
\limsup _{n=\infty} \frac{1-r_{n}}{t_{n}}=\rho_{1}, \quad \text { (iii) } \limsup _{n=\infty} \frac{t_{n}^{q+1}}{1-r_{n}}=\rho_{2}
$$

where $\rho_{1}, \rho_{2}$ are finite, it follows that

$$
\limsup _{n=\infty}\left|f\left(P_{n}\right)\right| \leqq \eta^{(2 / \pi) \arctan \left(\rho_{1}+2^{q} \rho \rho_{2}\right)-1} .
$$

The condition (i) prescribes that $E$ has metric density 1 (on one side) at $P$ and also limits the slowness with which $m(E \cdot A) / m A$ can approach 1 . The condition (ii) restricts $P$ to lie outside an angle one of whose sides is the radius to $P$ and the other some chord through $P$ in the upper half-plane. The condition (iii) allows the path of approach to be a tangent path but not of too high an order. Let $A_{n}$ be the arc on $|z|=1$ determined by $z=e^{i t}, 0 \leqq t \leqq 2 t_{n}$. Then using the same argument as that used several times already,

$$
\left|f\left(P_{n}\right)\right| \leqq \eta^{(2 / \pi) \arctan \theta\left(E, A_{n}, r_{n}\right)}
$$


and

so that

$$
\theta\left(E, A_{n}, r_{n}\right) \geqq \frac{\left(1+r_{n}\right) \frac{m\left(E \cdot A_{n}\right)}{m A_{n}}}{\frac{1-r_{n}}{\tan \left(t_{n} / 2\right)}+2^{q+2}\left(\frac{\tan \left(t_{n} / 2\right)}{t_{n}}\right)\left(\frac{t_{n}{ }^{q+1}}{1-r_{n}}\right)\left[\frac{1-\frac{m\left(E \cdot A_{n}\right)}{m A_{n}}}{\left(m A_{n}\right)^{q}}\right]},
$$

$$
\liminf _{n=\infty} \theta\left(E, A_{n}, r_{n}\right) \geqq\left(\rho_{1}+2^{q} \rho \rho_{2}\right)^{-1}
$$

which proves the theorem.

THEOREM 6. Let $f(z)$ be a bounded analytic function in $|z|<1$, with the boundary function $F(z)$. Let $E_{\mathrm{\epsilon}}$ be the set of those points on $|z|=1$ in the upper half-plane for which $|F(z)-\alpha| \leqq \epsilon$, for some fixed $\alpha, \epsilon>0$. Let $A$ be an arc of $|z|=1$ in the upper half-plane with one end point $P: z=1$, and let

$$
\limsup _{m A=0}\left[\frac{1-\frac{m\left(E_{\epsilon} \cdot A\right)}{m A}}{m A^{q}}\right] \leqq \rho, q>0,
$$

for some $\rho$ independent of $\epsilon>0$. Then if the sequence $\left\{P_{n}: r_{n} e^{i t_{n}}\right\}$ approaches $P: z=1$ so that

$$
\limsup _{n=\infty} \frac{t_{n}{ }^{q+1}}{1-r_{n}}=\rho_{2}
$$

is finite, it follows that $\lim _{n=\infty} f\left(P_{n}\right)=\alpha$.

A simple sufficient condition implying the hypotheses of the theorem is that $F(z)$ be continuous with limit value $\alpha$ on a point set $E$ in the upper halfplane such that

$$
\limsup _{m A=0}\left[\frac{1-\frac{m(E \cdot A)}{m A}}{(m A)^{q}}\right]
$$

is finite. We can assume that $\alpha=0$ and that $|f(z)| \leqq 1$. Suppose that the sequence $\left\{P_{n}\right\}$ contained a subsequence $\left\{P_{a_{n}}\right\}$ such that

$$
\lim _{n=\infty} f\left(P_{a_{n}}\right)=\beta \neq \alpha=0 .
$$

Since $\alpha=0$ is a metric cluster value of the first kind of $F(z)$ at $P$ of infinite or$\operatorname{der}, \lim _{z=1} f(z)=0$ if $z$ approaches $P$ keeping within some angle whose sides 
are chords of $|z|=1$ meeting at $P$. Then for large $n, P_{a_{n}}$ must be outside any given such angle. Thus the condition (ii) of Theorem 5 is satisfied for the sequence $\left\{P_{a_{n}}\right\}$ with $\rho_{1}=0$, so that

$$
\limsup _{n=\infty}\left|f\left(P_{a_{n}}\right)\right| \leqq \epsilon^{(2 / \pi) \arctan \left(2^{Q} \rho \rho_{2}\right)-1}
$$

for all $\epsilon>0$. Letting $\epsilon$ approach 0 ,

$$
\limsup _{n=\infty}\left|f\left(P_{a_{n}}\right)\right|=0
$$

which contradicts the hypothesis made that

$$
\lim _{n=\infty} f\left(P_{a_{n}}\right)=\beta \neq 0 \text {. }
$$

The theorem is then proved. It could evidently be generalized somewhat by having the constants concerned depend upon $\epsilon$. An application with $q=1$ is given by letting $z$ approach $P$ on a circle tangent to $|z|=1$ at $P$. The hypothesis that $P$ was the point $z=1$ was of course not essential, but made the statement of the theorem somewhat simpler.

HARVARD UNIVERSITY,

Cambridge, Mass. 